\title{
Expanding scroll rings and negative tension turbulence in a model of excitable media
}

\author{
S. Alonso, ${ }^{1, *}$ Ralf Kähler, ${ }^{2}$ A. S. Mikhailov, ${ }^{3}$ and F. Sagués ${ }^{1}$ \\ ${ }^{1}$ Departament de Química Física, Universitat de Barcelona, Av. Diagonal 647, 08028 Barcelona, Spain \\ ${ }^{2}$ Konrad-Zuse-Zentrum für Informationstechnik, Takustr. 7, 14195 Berlin, Germany \\ ${ }^{3}$ Abteilung Physikalische Chemie, Fritz-Haber-Institut der Max-Planck-Gesellschaft, Faradayweg 4-6, 14195 Berlin, Germany
}

(Received 9 October 2003; published 3 November 2004)

\begin{abstract}
Scroll waves in excitable media, described by the Barkley model, are studied. In the parameter region of weak excitability, negative tension of wave filaments is found. It leads to expansion of scroll rings and instability of wave filaments. A circular filament tends to stretch, bend, loop, and produce an expanding tangle that fills up the volume. The filament does not undergo fragmentation before it touches the boundaries. Statistical properties of such Winfree turbulence of scroll waves are numerically investigated.
\end{abstract}

DOI: 10.1103/PhysRevE.70.056201

PACS number(s): 05.45.Pq, 82.40.Bj

\section{INTRODUCTION}

Excitable media allow active propagation of individual waves and support regular wave patterns of rotating spirals or scroll rings. The Belousov-Zhabotinsky reaction [1], the populations of collective amoeba Dyctiostelium discoideum [2], and the cardiac tissue [3] are the most interesting examples of such media. Under certain conditions, regular wave propagation is replaced by a regime of wave chaos, loosely referred on what follows as turbulence. In the cardiac tissue, such regimes are known as fibrillation and they precede the cardiac death (see Refs. [4-6]). Therefore, investigations of turbulence in excitable media are of high practical importance. A characteristic property of the heart ventricle is that it is essentially three-dimensional (3D) and therefore the development of turbulence for scroll waves must be studied.

Rotating scroll waves have first been discovered in the chemical Belousov-Zhabotinsky reaction [7] and are also possible in the heart, see, e.g., Ref. [8]. In its cross section, a scroll wave has the shape of a spiral. Such spirals are stacked one upon another and rotate around a filament that occupies the center of a scroll. The filament may be straight or curved; it may also be closed into a ring or form knots. The dynamics of filaments plays a principal role in determining the evolution of three-dimensional wave patterns [9].

In two-dimensional (2D) excitable media, turbulence results from breakups of spiral waves which can either occur on local heterogeneities $[10,11]$ or take place spontaneously, due to intrinsic dynamical instabilities of such patterns [12-15]. Breakups of steadily rotating spiral waves are, however, absent and the development of turbulence is prohibited in weakly excitable systems [16], where the rotation period of a spiral wave is much larger than the refractory time and the medium completely recovers after passage of each next excitation pulse. In three-dimensional systems, transition to turbulence may also proceed through wave breakups and thus be similar to the phenomena in two dimensions [17]. An interesting question is whether other, principally different

\footnotetext{
*Author to whom correspondence should be addressed. Email address: s.alonso@qf.ub.es
}

routes to turbulence are further possible in three dimensions.

This question has been raised by Winfree who, based on the analysis of cardiological data, has proposed that fibrillation is often not accompanied by any wave breakups [18]. Instead, it develops through a disorderly dynamics of filaments that "snake about" in the medium. In a recent publication [19], we have suggested that this kind of spatiotemporal chaos should be called Winfree turbulence of scroll waves to commemorate profound contributions made by Winfree in studies of pattern formation in excitable media.

The turbulence in weakly excitable media is based on the effect of negative tension of scroll wave filaments. Already some time ago, it has been noticed that scroll rings may not only contract $[20,21]$, but also expand in the parameter regions corresponding to weak excitability $[16,22,23]$. It has been conjectured that such expansion must imply an instability of straight filaments and their complex dynamics [16,23]. Subsequently, the effect of negative filament tension has been confirmed in fully 3D numerical simulations $[9,24]$ and has been demonstrated by the direct numerical stability analysis of straight untwisted scrolls $[25,26]$. Moreover, it has also been found in models of cardiac tissue [27,28], thus emphasizing its relevance for heart fibrillation.

Previous numerical investigations [9,24,27-29] have shown that the negative-tension instability leads to irregular dynamics of filaments and to the emergence of complex wave patterns. In our recent brief report [19], we have shown some results of extensive large-scale simulations of this kind of turbulence in the Barkley model and have also shown that this form of spatiotemporal chaos can be controlled by application of weak nonresonant forcing. The aim of the present paper is to provide a full account and the detailed analysis of the simulations in absence of external forcing. The phenomena related to external forcing and the respective theoretical description shall be a subject of a separate publication [30].

We choose for our simulations the Barkley model [31], where spontaneous breakup of spiral waves is not possible, and we restrict ourselves to parameter regions were meandering of spiral waves and the respective additional instability of scroll filaments $[25,26,32]$ are excluded. Our simulations indicate that negative tension of filaments is found in a large part of the low-excitability region and therefore this 
turbulence is generic. Because of the instability, the filament stretches up and forms a growing complex tangle. This behavior resembles the development of hydrodynamical turbulence through the expansion of flow vortices in 3D fluids. The growing filament remains singly connected and budding of new small scroll rings does not take place. The filament undergoes fragmentation only when it touches the (no-flux) boundaries of the medium. The statistical analysis of the developed Winfree turbulence shows rapid decay of spatial and temporal correlations, characteristic for spatiotemporal chaos.

In the next section, we present the mathematical model and describe numerical methods used for its simulations. To identify the parameter region, where negative tension of filaments is observed, a series of simulations of collapsing or expanding scroll rings in the assumption of radial symmetry (and thus effectively two-dimensional) is performed in Sec. III. Full 3D simulations and the analysis of statistical properties are presented in Sec. IV. The paper ends with the conclusions and the discussion of obtained results.

\section{MODEL AND NUMERICAL METHODS}

Numerical simulations were performed with the Barkley model [31] which is relatively simple and is known to yield wave patterns generic for excitable media. The dimensionless reaction-diffusion equations of this model are

$$
\begin{gathered}
\partial_{t} u=D_{u} \nabla^{2} u+\frac{1}{\epsilon} u(1-u)\left(u-\frac{v+b}{a}\right), \\
\partial_{t} v=D_{v} \nabla^{2} v+u-v .
\end{gathered}
$$

Here $u$ and $v$ are the activator and the inhibitor concentrations, $D_{u}$ and $D_{v}$ are diffusion constants of the activator and the inhibitor; and $\epsilon$ is the ratio of their temporal scales. The parameters $a$ and $b$ specify the activator kinetics, with $b$ effectively controlling the excitation threshold of the system. Note that the model (1) with $D_{v}=0$ can also be interpreted as describing the cardiac tissue (in this case the local coupling for the activator variable is of electrical origin). If the parameters $a$ and $b$ are chosen positive, the model corresponds to an excitable medium if $a<1+b$ and to a bistable medium otherwise. Later we always take $\epsilon=0.02$ and $D_{u}=1$.

In our numerical simulations, Eqs. (1) were integrated using the explicit Euler algorithm on a regular rectangular grid inside a cube of linear size $L$ (varying from 60 to 80 in different simulations) and no-flux boundary conditions on its planes. The time and coordinate steps were chosen depending on the model parameters: typically, three-dimensional simulations are conducted using $\Delta x=0.4$ and $\Delta t=0.012$ and two-dimensional using $\Delta x=0.2$ and $\Delta t=0.008$. To initiate a scroll ring, the procedure described in Ref. [33] has been employed: First, an expanding spherical wave was created by applying a superthreshold perturbation in the center of the medium. Then we waited until the waves reaches a desired radius and, at that moment, reseted half of the medium back to the original rest state. In this manner, a spherical cup with a growing edge was produced, which soon evolved into a scroll ring.
Since filaments are important for the characterization of scroll wave patterns, their locations should be computed. In the literature, several different methods [31,34-36] have been used for identifying wave filaments in numerical simulations. In the method, proposed in Ref. [34], the filament is defined as the line formed by the intersection of two surfaces given by equations $u(\mathbf{r}, t)=0.05$ and $v(\mathbf{r}, t)=a / 2-b$ (the latter condition ensures that $\partial_{t} u=0$ on the respective surface when diffusion is negligible). In our simulation, a filament was defined as a set of points where both the activator and inhibitor concentrations lie inside the intervals $[1 / 2$ $-\Delta u, 1 / 2+\Delta u]$ and $[a / 2-b-\Delta v, a / 2-b+\Delta v]]$, with $\Delta u$ $=0.075$ and $\Delta v=0.05$. With this definition, the filament is not a line, but a set of points. Although this method is not as accurate as those cited earlier, it allows a fast computation and it is reliable enough to capture generically the destabilization mechanism we are interested in. Note also that this filament localization mechanism is not going to be used in the statistical characterization of the disorganized pattern of wave propagation.

The scroll rings drift along their symmetry axis (which was vertical in our simulations). Because of this drift, they reach the boundaries and die out there. Since we wanted to see the evolution of a ring over a long time, but could not significantly increase the linear size of the medium, "moving boundaries" along the vertical direction were implemented for scroll rings with a strong vertical drift. At each time moment, the center of mass for the ring filament has been calculated. Then, we removed one horizontal slice of grid points in the lattice and added it up in the direction of the drift, so that the filament was kept in the center of the threedimensional medium.

To speed up the simulations, we had to choose relatively large values for the time and coordinate steps in the integration scheme. By comparing with finer discretizations we confirmed that some quantitative observations of the propagating waves are slightly modified, but such changes are small (for instance frequency of spiral waves do not differ by more than a 5\%) and do not alter significantly the main conclusions of our analysis. To eliminate the grid effects that become otherwise visible, giving rise to unrealistic too symmetric shapes, weak white noise $\eta(x, t)$ with correlation $\left\langle\eta(x, t) \eta\left(x^{\prime}, t^{\prime}\right)\right\rangle=2 d \delta\left(x-x^{\prime}\right) \delta\left(t-t^{\prime}\right)$ and intensity $d=1.25$ $\times 10^{-5}$ was always added to the activator evolution equation. This weak additive noise practically does not modify the propagation properties, for example the frequencies of wave rotation are modified by less than a $1 \%$.

In addition to full 3D simulations, reduced 2D simulations for scroll rings were also performed in some cases. Assuming axial symmetry of a scroll ring with respect to its vertical axis, one can write the Laplacians in cylindrical coordinates and omit the derivatives with respect to the polar angle. As a result, the simulation becomes effectively two-dimensional, because only the radial and the vertical coordinates should be considered. Originally, this simplified integration has been used in Ref. [22]. For expanding scroll rings, that soon lose the initial axial symmetry due to the negative-tension instability of the filament, this integration method is only applicable at the initial evolution stage, to determine the expansion rate. 
Finally, numerical simulations of spiral waves in the model (1) were also performed in our study. Their aim was to determine the boundaries in the parameter plane $(a, b)$ where meandering of spiral waves begins and where the medium becomes subexcitable, so that the rotation period of spiral waves diverges. The explicit Runge-Kutta algorithm has been used for the integration of the pure $2 \mathrm{D}$ and the reduced 3D simulations.

\section{EXPANSION AND CONTRACTION OF SCROLL RINGS}

As follows from the general theory, evolution of nontwisted large scroll rings should be described by the following equations $[20,23]$ :

$$
\frac{d R}{d t}=-\frac{\alpha}{R}, \quad \frac{d Z}{d t}=\frac{\beta}{R},
$$

where $R$ is the radius of the ring and $Z$ is its coordinate along the symmetry axis. These equations also hold locally for curved nontwisted filaments, if $R$ is chosen as the local curvature radius. The parameter $\alpha$ represents the filament tension coefficient of the medium. If $\alpha>0$, scroll rings contract. On the other hand, negative filament tension $(\alpha<0)$ implies expansion of scroll rings. The parameter $\beta$ specifies the rate of the ring drift along its symmetry axis. Depending on the medium parameters, it may also change its sign.

It is worth recalling here that Eq. (2) earlier also applies to electrically forced spiral waves after changing curvature by the intensity of the electric field. In this respect the transition between expanding and contracting scroll rings translates in to a reversal in the direction of the drifting spiral wave [37].

When diffusion constants of all involved species are equal (that is, $D_{u}=D_{v}=D$ in the activator-inhibitor model), the vertical drift disappears $(\beta=0)$ and the filament tension coefficient becomes $\alpha=D$ (see Ref. [38]). Thus scroll rings in such media always undergo collapse. General analytical estimates for the coefficients $\alpha$ and $\beta$ are available in the phenomenological kinematic theory of scroll waves $[16,23,39]$. According to these estimates, scroll rings in media with $D_{u}>D_{v}$ and sufficiently weak excitability (close to the boundary of subexcitable regime) are expected to expand.

Available numerical simulations provide examples of both contracting [40] and expanding [22] scroll rings. It should be noticed that contracting scrolls have been more often considered. As we shall see, the parameter region for expanding scroll rings is actually also quite broad. However, it lies closer to the subexcitability boundary and corresponds to the spirals with relatively large wavelengths which is less convenient for numerical simulations.

We have numerically computed the values of the filament tension coefficient $\alpha$ in the entire parameter region of the Barkley model where rigidly rotating spiral waves are observed. This has been done by determining the expansion (contraction) rates of scroll rings from numerical simulations assuming radial symmetry (and thus effectively twodimensional, see the previous section). The results are presented in Fig. 1. We see that the region ES with negative

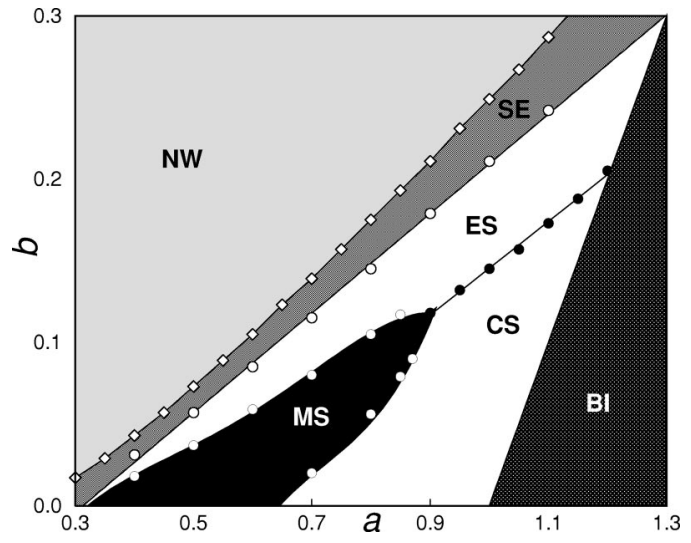

FIG. 1. State diagram of the Barkley model. The parameter regions are NW, no waves (decay of pulses), SE, subexcitable (shrinking of waves with free ends), BI, bistability, MS, meandering spirals, CS, collapsing scroll rings, and ES, expanding scroll rings. The values of other parameters are $\varepsilon=0.02, D_{u}=1$, and $D_{v}=0$. Reproduced from Ref. [19].

filament tension $(\alpha<0)$ occupies a portion of the parameter plane which is comparable in its size with the region where contraction $(\alpha>0)$ of filaments is observed.

The negative tension region corresponds to systems with lower excitabilities (larger values of the model parameter $b$ specifying the excitation threshold). It lies near the boundary of the subexcitable region SE. This boundary is defined by the condition that the rotation period of spiral waves diverges (i.e., becomes formally infinite) there. Above this boundary, a broken flat wave contacts; below it, the tip grows and a spiral wave is produced [16]. Note that, sufficiently close to this boundary, the rotation period of spiral waves is larger than the recovery time. The medium undergoes then complete recovery after passage of each next wave, so that interactions between the subsequent waves are absent and rigid rotation of spiral waves takes place.

Figure 1 also displays the region (MS) where meandering of spiral waves is observed. In this region, computation of the filament tension coefficient using the effectively $2 \mathrm{D} \mathrm{nu}-$ merical scheme is not pursued.

It should be noted that spiral waves and scroll rings are also possible inside the region $\mathrm{BI}$, where the medium is bistable. However, we have not extended our analysis into this region since we were mainly interested in the case of excitable kinetics.

Figure 2 shows the dependence of the coefficients $\alpha$ and $\beta$ on the parameter $b$ specifying the excitation threshold in the Barkley model once fixing the remaining parameters. We see that the filament tension coefficient $\alpha$ varies between approximately -2 and 1 , changing its sign on the boundary separating expanding and contracting scroll rings. The coefficient $\beta$, specifying the rate of the vertical drift, remains positive in the entire interval of $b$. Remarkably, the drift rate has a clear maximum near a transition from expansion to contraction (where $\alpha=0$ ), in such a way that the modulus of the velocity of the scroll ring changes monotonously [41].

Though most of the simulations reported in this paper have been performed assuming that diffusion of the inhibitor variable is absent (a feature which is characteristic for the 


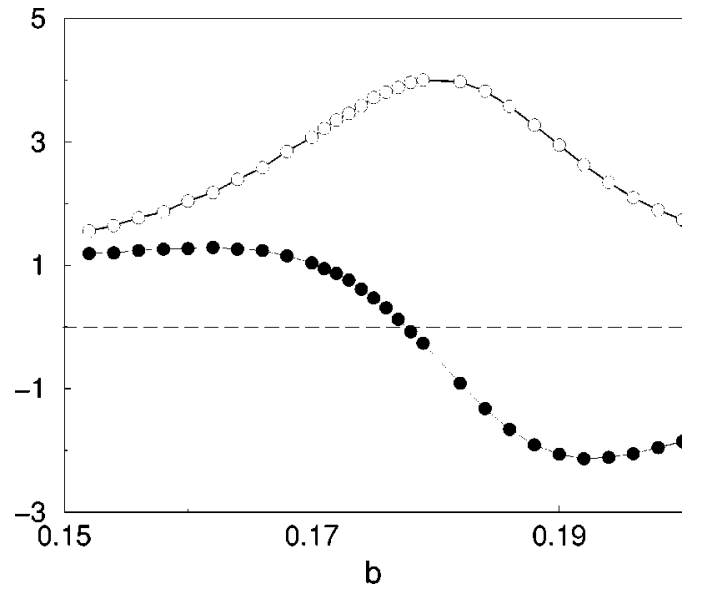

FIG. 2. Dependence of the filament tension coefficient $\alpha(\mathbf{O})$ and the vertical drift coefficient $\beta(\bigcirc)$ on the parameter $b$ at $a=1,1$. Other parameters are the same as in Fig. 1. Numerical integration with $\Delta x=0.2$ and $\Delta t=0.008$.

cardiac tissue or for chemical reactions in gels [42]), we have also done some simulations to study the behavior of scroll rings in excitable media where both components are diffusing. Figure 3 displays the dependence of $\alpha$ and $\beta$ on the inhibitor diffusion constant $D_{v}$ in the parameter region where expansion of scroll rings is observed in absence of the inhibitor diffusion. We see that the filament tension coefficient changes its sign and becomes positive already at relatively slow inhibitor diffusion $\left(D_{v} \approx 0.1\right)$. When both diffusion constants coincide $\left(D_{u}=D_{v}=1\right)$, the vertical drift disappears $(\beta$ $=0$ ) and the filament tension coefficient becomes equal to the diffusion constant $(\alpha=1)$. Because the diffusion constants of all reacting species are usually close for liquid solutions (for instance, $D_{\text {cat }} / D_{\mathrm{BrO}}=0.6$ for the Belousov-Zhabotinsky reaction [43]), expansion of scroll rings and the related phenomenon of the Winfree turbulence here analyzed should not be generally expected for such chemical systems.

Finally, Fig. 4 shows the dependence of the drift coefficient $\beta$ on the parameters $a$ and $b$ in the regions ES and CS

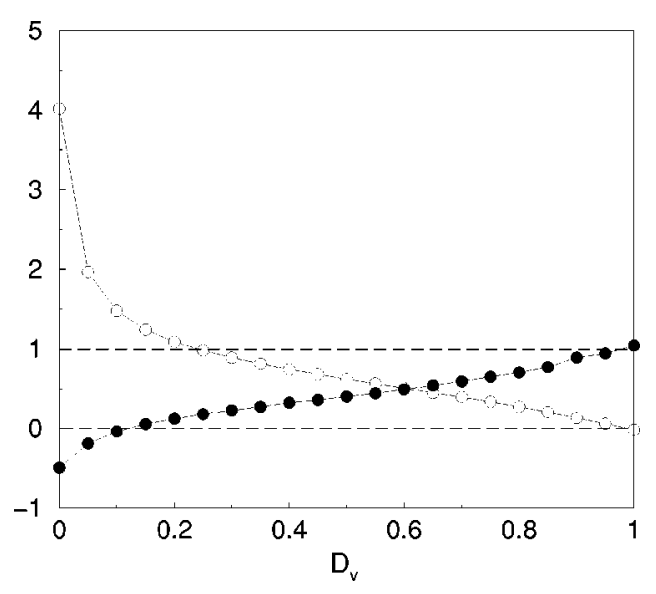

FIG. 3. Dependence of the filament tension coefficient $\alpha(\mathbf{O})$ and the vertical drift coefficient $\beta(\bigcirc)$ on the diffusion constant $D_{v}$ of the inhibitor at $a=1,1$ and $b=0.18$. Other parameters are the same as in Fig. 2. occupied by expanding and contracting scroll rings. From these numerical simulations, we conclude that the vertical drift becomes very weak and may change its direction close to the boundary of the region ME where meandering of spiral waves is observed.

\section{DEVELOPMENT AND STATISTICAL PROPERTIES OF WINFREE TURBULENCE}

The evolution of an expanding scroll ring leading to fully developed negative tension turbulence is shown in Fig. 5 (see Ref. [44] for the visualization of the complete movie). For visualization, spatial regions where the local activator field $u$ exceeds 0.9 are displayed by semi-transparent lightgray color. The $3 \mathrm{D}$ visualization was realized using the AMIRA software package (Template Graphics Software). Initially, the scroll ring is circular [Fig. 5(a)]. However, perturbations in the central region soon develop and the wave pattern in this region becomes irregular [Fig. 5(b)]. The irregular wave pattern expands [Figs. 5(c) and 5(d)] and fills up the whole medium [Figs. 5(e) and 5(f)].

To better understand this process, the respective evolution of the scroll wave filament can be considered (Fig. 6) and, Ref. [45]. During the initial stage, the expanding filament remains circular [Fig. 6(a)]. After a few tens of wave rotations, the filament begins to wrinkle [Fig. 6(b)]. As the wrinkles develop, the filament ceases to be planar and some modulation along the vertical axis is also seen. We have noticed that the characteristic length scale of the filament instability in our simulations was numerically close to the spatial period of waves emitted by a scroll. The wrinkles were not stationary, but rather moving along the filament. Subsequently, the modulation grows [Fig. 6(c)] and the filament forms a complex expanding tangle [Figs. 6(d) and 6(e)]. After the filament touches the (no-flux) boundaries of the medium, its fragmentation occurs and many disconnected filament parts are produced [Fig. 6(f)].

The absence of spontaneous fragmentation of the filament is a characteristic property of the negative tension turbulence. In a scroll breakup, new pieces of filaments could bud up at the break points $[17,27]$, which we have not seen in our simulations. The breakup of scroll waves is closely related to the respective phenomenon for spiral waves in two dimensions. It should be noted that, in the considered Barkley model, breakups of spiral waves have not been found too. Moreover, the negative tension instability of filaments is observed relatively low medium excitabilities, where the medium has enough time to (almost) recover after passage of each next wave. Under these conditions, spontaneous breakups of waves cannot generally be expected. Apparently, there is some kind of repulsion between the filaments [46].

The fragmentation, however, occurs when the filament touches a boundary of the medium. The no-flux boundary condition effectively implies that a collision of a filament with its mirror image is taking place. One may ask why such a fictitious collision leads to the filament breakup, though real collisions between different parts of filaments lead only to mutual avoidance of filaments. This behavior may be related to the special symmetry present when a filament "col- 

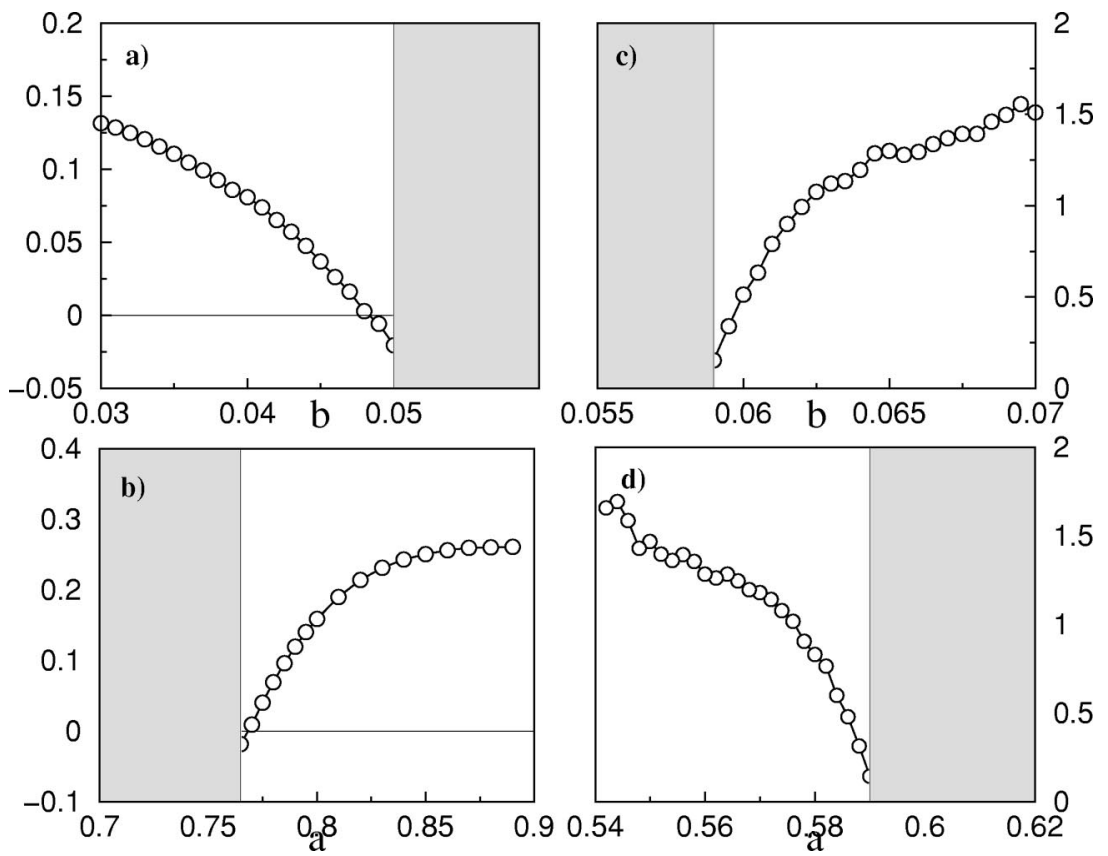

FIG. 4. Dependence of the vertical drift coefficient $\beta$ on the parameters $a$ and $b$ near the boundary of the meandering region (gray area) for $(\mathrm{a}, \mathrm{c}) \quad a=0.59$, (b,d) $b=0.059$. Other parameters are the same as in Fig. 2. lides" with its mirror image [34,46]. It is known that, in order to undergo reconnection needed for fragmentation, the approaching filament parts must lie in the same plane [47]. Repulsive interactions between real filaments are probably preventing such an arrangement. But when a filament collides with its own mirror image, this arrangement is imposed by the symmetry and is thus nonavoidable.

Numerical simulations of instabilities and irregular wave dynamics due to the effect of negative tension have previously been performed by Biktashev et al. [9,24], by Gray and Jalife [28], by Chavez et al. [29], and by Fenton et al. [27]. In these simulations, a linear filament spanning the medium or a quarter of a scroll ring were chosen as initial conditions. It was noticed that the thickness of the medium should exceed a certain threshold in order that the negative tension instability of linear filaments develops. These simulations have also shown that fragmentation of the filament only begins when it touches the boundary plane. Our present numerical simulations are performed for substantially larger systems, over much longer times and with the initial conditions representing full scroll rings. Indeed, the largest linear dimension of the medium in such earlier simulations was of the order of the wavelength lambda of the scroll wave. In contrast to this, the linear size of the medium in our studies is more than four times larger than the wavelength [e.g., in Fig. 5 we have $\lambda=13.4]$. This corresponds to an increase by two orders of magnitude in terms of the simulated medium volume. Furthermore, we have followed evolution of wave patterns in the turbulent regime for a total time of 200 rotation periods, whereas in the previous investigations only up to 26 rotations were seen in Biktashev et al. [9] and even less in Fenton et al. [27].

In our study, a filament has been defined as a set of grid points where both the activator and the inhibitor concentrations lie within a narrow prescribed intervals (see Sec. II). The points, forming a filament, have been computed at each time step and thus the gradual evolution of this object could be observed. It should be noticed that our conclusions about the connectivity of the filament and its fragmentation are based on visual monitoring of the development. Though the evidence is already compelling (see Ref. [45]), a formal proof would require more refined data processing and is not yet provided.

To quantify the evolution, several filament properties have been computed. If a filament is defined by a set of $N$ grid points $\left(x_{i}, y_{i}, z_{i}\right)$, its center of mass $(X, Y, Z)$ can be determined as

$$
\begin{aligned}
& X(t)=\frac{1}{N} \sum_{i=1}^{N} x_{i}(t), \\
& Y(t)=\frac{1}{N} \sum_{i=1}^{N} y_{i}(t), \\
& Z(t)=\frac{1}{N} \sum_{i=1}^{N} z_{i}(t) .
\end{aligned}
$$

Note that $Z(t)$ yields the vertical position of the filament. The mean radius $R$ of the filament in its horizontal projection is given by

$$
R=\frac{1}{N} \sum_{i=1}^{N} \sqrt{\left(x_{i}-X\right)^{2}+\left(y_{i}-Y\right)^{2}} .
$$

The mean radial dispersion $\Delta R$ in the horizontal plane is defined as

$$
\Delta R^{2}=\frac{1}{N} \sum_{i=1}^{N}\left[\sqrt{\left(x_{i}-X\right)^{2}+\left(y_{i}-Y\right)^{2}}-R\right] .^{2}
$$

Moreover, the total length $L$ of the filament, considered proportional to the number of points $N$, can be conveniently defined as $L(t)=\xi \Delta x N(t)$, where $\Delta x$ is the grid step, and $\xi$ is 

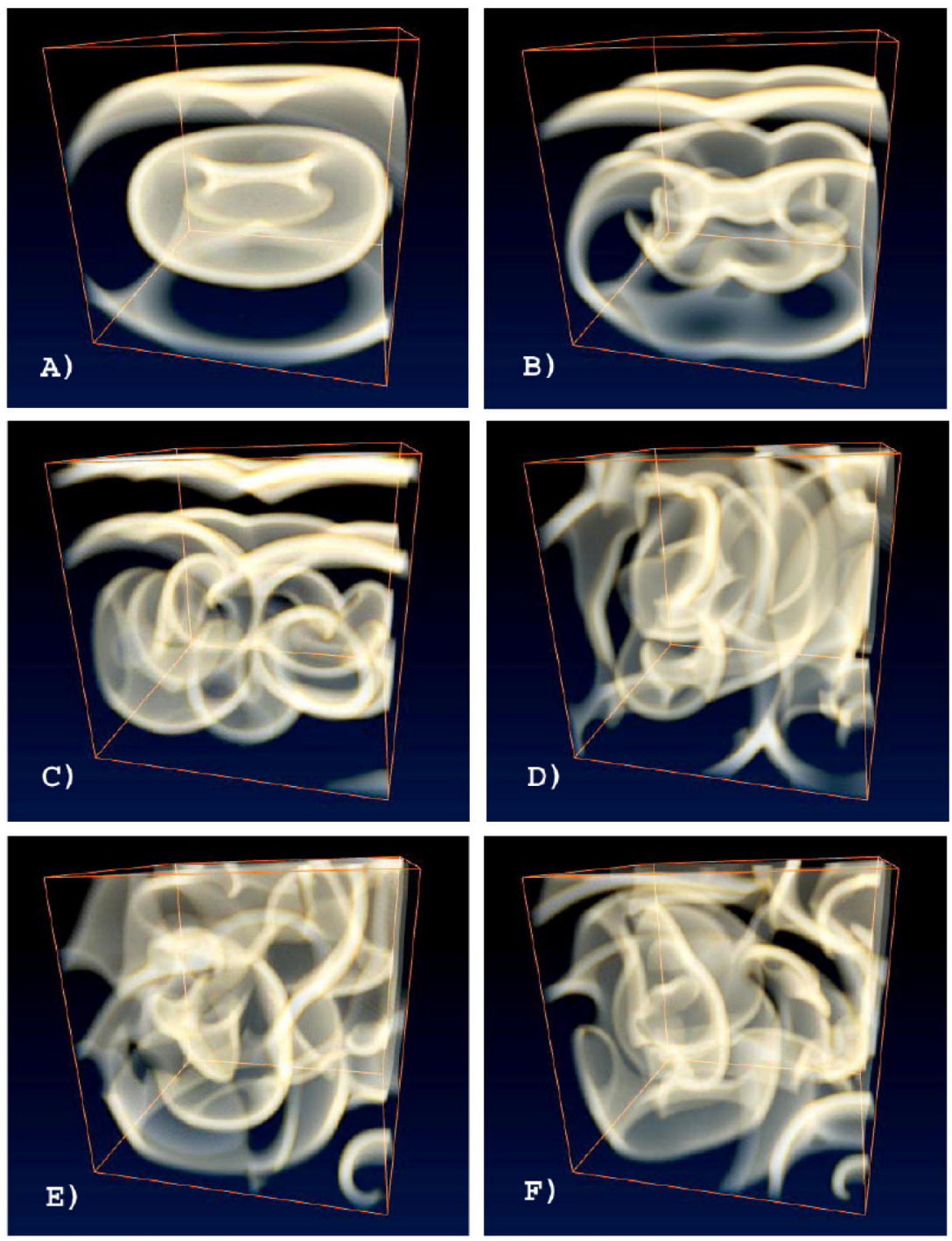

FIG. 5. Develoment of negative tension turbulence. Subsequent snapshots of wave patterns in the medium of size $60 \times 60 \times 60$ are shown at the time moments (a) 5, (b) 80, (c) 120, (d) 240, (e) 320, and (f) 395. Semitransparent visualization of the activator distribution is chosen. The parameters are $a=1.0$ and $b=0.16$; other parameters are the same as in Fig. 1. Numerical integration on a cubic grid with fixed steps $\Delta x=0.4$ and $\Delta t$ $=0.012$. a prefactor calculated from the very initial state when the filament is completely circular and it is defined as $\xi$ $=2 \pi R_{0} / N_{0} \Delta x$.

Figure 7 displays the evolution of these properties as the expanding ring undergoes an instability leading to turbulence. We see that during the initial expansion stage, when the filament remains approximately circular (approximately until $t=75$ ), its length [Fig. 7(a)] and its radius [Fig. 7(b)] are slowly increasing, but the radial dispersion remains small [Fig. 7(c)]. The shape destabilization and the development of turbulence $(t>100)$ are marked by an increase in the radial dispersion and by a strong growth of the filament length. In parallel, the vertical motion of the filament is slowed down and stops in the turbulent regime where the filament already has a globular tangled shape without any preferred orientation [Fig. 7(d)].

Figure 8(a) shows the evolution of the filament length on a longer time scale. Pronounced oscillations in this property are due to the filament pieces leaving the medium through its boundaries. Additionally, Fig. 8(b) displays the mean activator concentration $\langle u\rangle$, averaged over the whole medium. Note that this property remains approximately constant in the tur- bulent regime, so that the excitation waves are filling the whole available volume.

If the wave turbulence here described represents a form of spatiotemporal chaos, it should be revealed in its statistical properties. To analyze them, spatial and temporal correlation functions $S(\rho)$ and $S(\tau)$ of the activator field $u(\mathbf{r}, t)$ in the turbulent states have been computed. These functions were defined as

$$
S(\rho)=\frac{1}{\left\langle\delta u^{2}\right\rangle}\langle\delta u(\mathbf{r}+\rho, t) \delta u(\mathbf{r}, t)\rangle
$$

and

$$
S(\tau)=\frac{1}{\left\langle\delta u^{2}\right\rangle}\langle\delta u(\mathbf{r}, t+\tau) \delta u(\mathbf{r}, t)\rangle,
$$

where $\delta u(\mathbf{r}, t)=u(\mathbf{r}, t)-\langle u\rangle$.

For the spatial correlation function $S(\rho)$, a spatial average over the entire system volume was taken. Additionally, averaging over eight turbulent patterns, corresponding to different times, was performed. The temporal correlation function $S(\tau)$ was computed by taking time averaging of data at a 


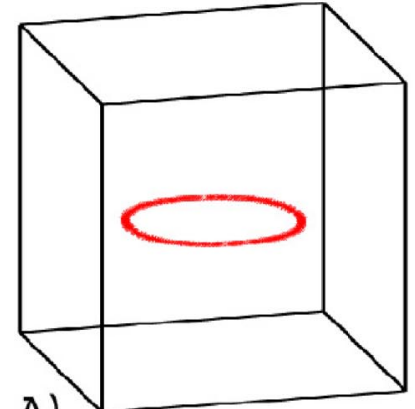

A)
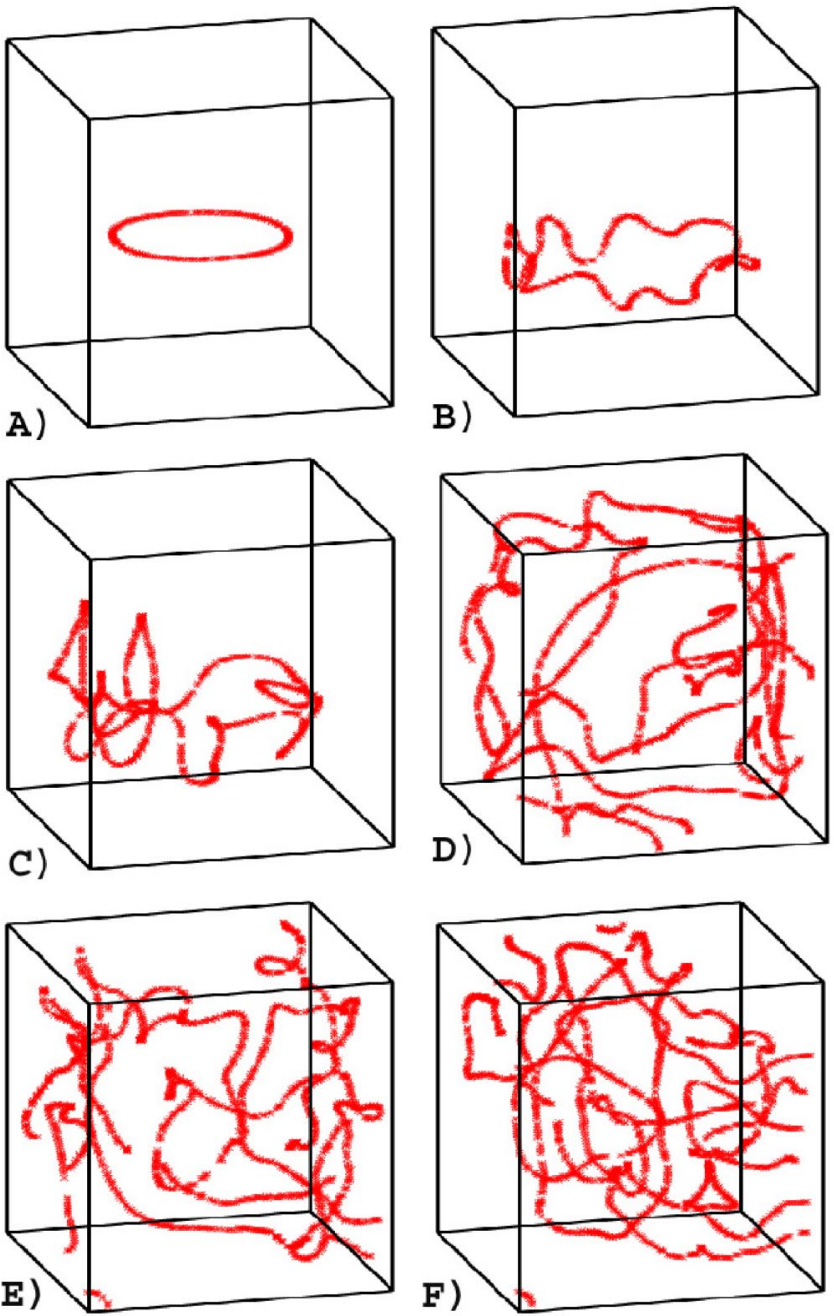

FIG. 6. Evolution of the filament. The data is taken from the same simulation and at the same time moments as in Fig. 5.

given coordinate point in the medium. Additionally, averaging over eight different points of the same realization was performed.

As seen in Fig. 9, the spatial correlation function is rapidly falling to zero, as expected for spatiotemporal chaos. The characteristic correlation length is close in its magnitude to the wavelength of a scroll wave in the considered excitable medium.

The temporal correlation function $S(\tau)$, depicted in Fig.10(a), is also vanishing for long time intervals in the turbulent regime. However, it also shows damped oscillations, indicating that the turbulent regime corresponds to irregular cyclic excitation of the medium elements with a certain mean characteristic time $T_{0}$. (cf. Ref. [48] in the cardiological context) The presence of this characteristic oscillatory time scale is also clear in the power spectrum $S(\omega)$, shown in Fig. 10(b). Additionally, we have shown in Fig. 10 the correlation function and the power spectrum for a regular pattern of a rotating spiral wave in the $2 \mathrm{D}$ medium with the same parameters. The correlation function is periodic, with the period corresponding to the rotation period $T_{S}$ of the spiral wave. Note that the characteristic oscillatory time scale $T_{0}$ for the turbulent regime is somewhat shorter than the rotation period $T_{s}$ of spiral waves in the same medium (a similar feature was also observed in Ref. [24]).

We have performed a series of 3D simulations of the turbulent regimes, to analyze the statistical properties at different values of the parameter $b$ that specifies the excitation threshold in the Barkley model. In Fig. 11, the characteristic oscillatory time scale $T_{0}$ of turbulence is plotted as function of $b$ together with the rotation period $T_{s}$ of spiral waves. As the boundary of the subexcitable region SE is approached, the rotation period of spiral waves strongly increases and this is paralleled by an increase of the turbulence time scale. Additionally, we have plotted in the same figure the refractory and the recovery times for the same excitation thresholds (the refractory time is defined as the minimum time to allow next pulse propagation, whereas the recovery time is estimated from the numerical simulations as the time the system needs for the total recovery). Near the boundary of the subexcitable medium, the characteristic timescale $T_{0}$ of turbulence becomes significantly higher than the recovery time. This means that medium completely recovers after each next local excitation and the subsequent propagating waves cannot feel one another. This again clearly indicates that the considered kind of turbulence is principally different from spiral wave breakup.

Figure 12 shows the dependence of several properties of the turbulent dynamics on the excitability of the system specified by the parameter $b$. As the parameter $b$ increases and the medium becomes less excitable, the height of the main maximum $S\left(T_{0}\right)$ of the temporal correlation at the characteristic time $T_{0}$ decreases [Fig. 12(a)] and the respective maximum in the power spectrum becomes more broad [Fig. 12(b)]. Hence, local oscillations become more irregular for less excitable media. The difference between the mean activation $\langle u\rangle$ in the $3 \mathrm{D}$ disorganized state and the respective mean activation for spiral waves in the $2 \mathrm{D}$ medium with the same parameters is displayed in Fig. 12(c). The increase of this difference with the parameter $b$ indicates that, at low excitability, the waves are filling the medium in the turbulent regime more densely than the respective spiral wave pattern. Finally, we show in Fig. 12(d) the relative difference between the characteristic time scale $T_{0}$ in the state of wave turbulence and the rotation period $T_{s}$ of spiral waves. Similarly their difference increases when excitability decreases.

\section{DISCUSSION AND CONCLUSIONS}

Our numerical investigations reveal that turbulence of scroll waves represents a typical form of spatiotemporal chaos in reaction-diffusion systems with weak or absent inhibitor diffusion. The turbulence associated with the negative tension of filaments is found in a broad parameter region next to the boundary of the subexcitable regime. Its mechanism is principally different from the breakup of spiral waves, responsible for the onset of turbulence in twodimensional excitable systems. Instead, it shows a significant similarity with the processes characteristic for hydrodynamical turbulence in three spatial dimensions. In hydrodynamics, the turbulence is generated by vortices whose filaments 

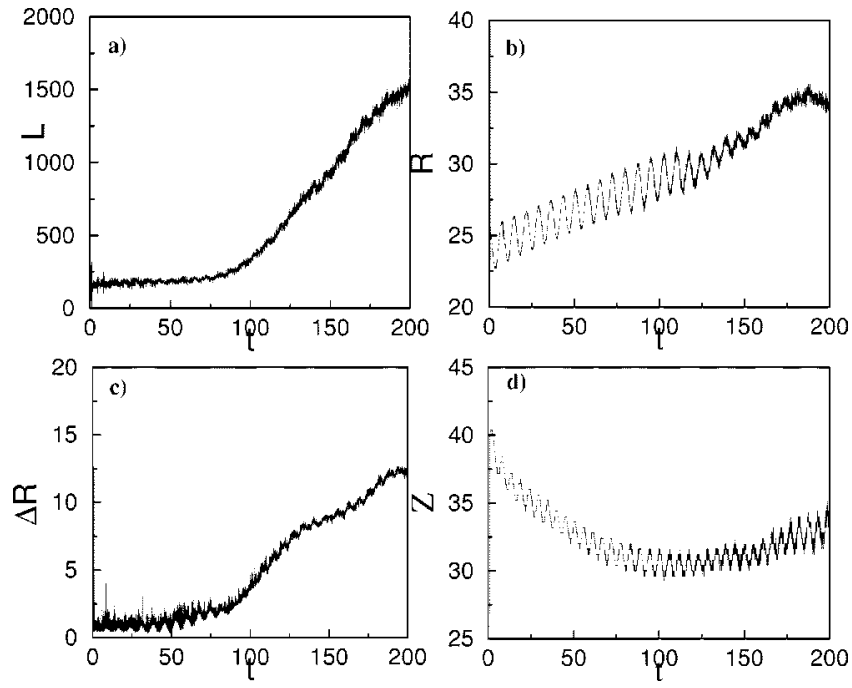

FIG. 7. Time dependences of (a) the total filament length, (b) the mean filament radius, (c) the radial dispersion, and (d) the vertical position of an expanding scroll ring in the medium of size $60 \times 60 \times 60$. The parameters are $a=1.1$ and $b=0.19$; other parameters the same as in Fig. 1.

stretch, take irregular form, and make loops. In excitable media, analogous behavior is found by us for the filaments of scroll waves. There is also an important difference between the two kinds of turbulence. The equations of hydrodynamics possess the Galilean invariance, so that a local pattern can be entrained into a larger scale flow. Consequently, the fluid turbulence is characterized by a hierarchy of vortices of different sizes, where the smaller vortices belong to the flow patterns of the larger ones. This invariance is absent in reaction-diffusion systems, where the rest coordinate frame is well defined.

In our simulations for the Barkley reaction-diffusion model, we have always seen that the expanding filament remains singly connected even when the state of developed turbulence is reached. This suggests that spontaneous budding of new scroll rings does not take place and the entire medium is still occupied in the turbulent regime by a single vortex (scroll ring) which is deformed into a very complex
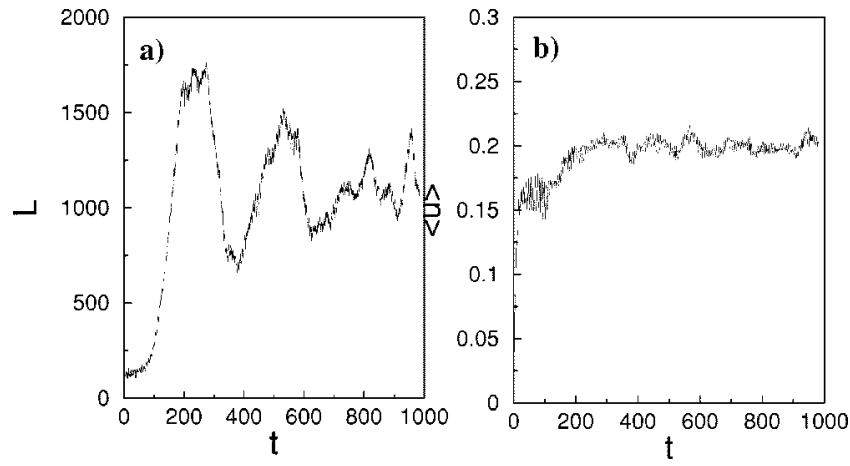

FIG. 8. Time dependences of (a) the total filament length and (b) the mean activator value over a longer time interval. The parameters are $a=1.1$ and $b=0.21$; other parameters the same as in Fig. 1 .

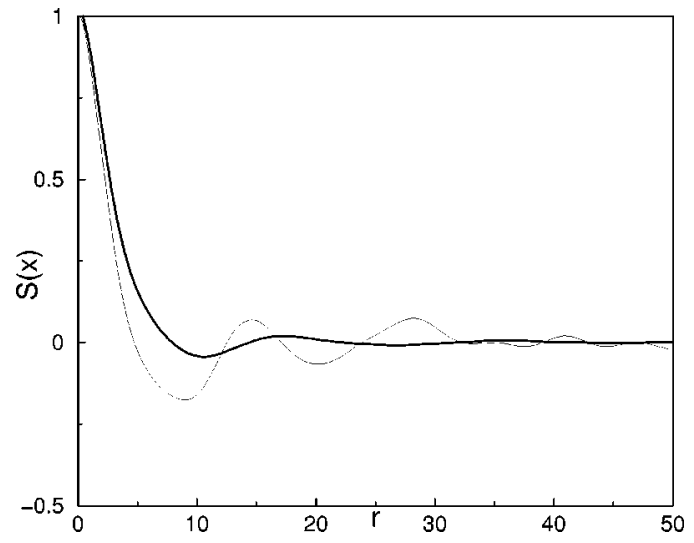

FIG. 9. Spatial correlation function of negative tension turbulence. Averaging over eight different snapshots in the turbulent regime. The thin line shows the spatial correlation function for a spiral wave in the 2D medium. The same parameters as in Fig. 8.

tangle. Only when the filament touches a boundary of the medium, its fragmentation occurs.

The negative tension instabilities of filaments is also similar to the static instability of interfaces, which is responsible for fingering in crystal growth and the formation of labyrinthine patterns in some activator-inhibitor systems. The difference is that the filaments represent rotation centers for the waves and, when a complex evolving pattern is formed by them, this corresponds to an irregular dynamical regime. Though we have not yet performed a detailed study of the negative tension turbulence in terms of Lyapunov exponents and embedding dimensions, the computed correlation functions already yield evidence of the chaotic behavior inherent in such irregular wave patterns.

Winfree claimed that irregular dynamics of scroll filaments should be of fundamental importance for heart fibrillation and the instances of sudden cardiac death. Our field of expertise is far from cardiology. Nonetheless, we can notice that this suggestion is to a certain extent supported by our numerical findings. The turbulence develops from straight
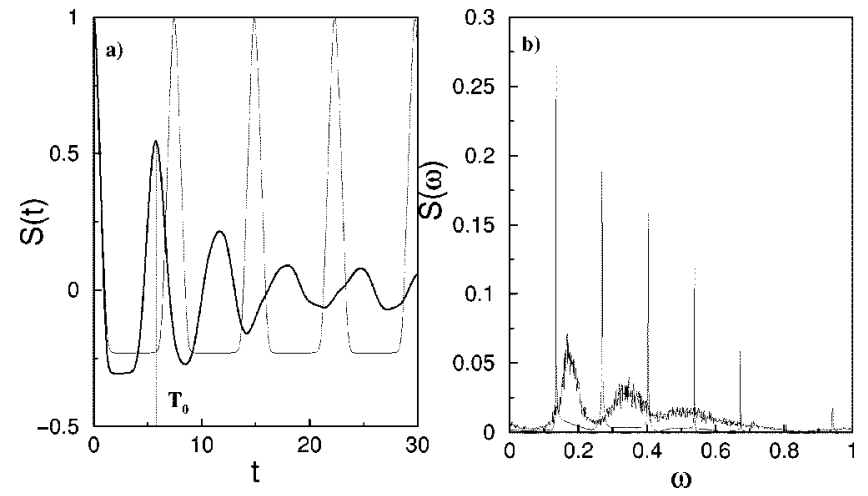

FIG. 10. Temporal correlation function (a) and power spectrum of negative tension turbulence. Averaging over eight different spatial points. The thin lines show the temporal correlation function and the power spectrum for a spiral wave in the $2 \mathrm{D}$ medium. The same parameters as in Fig. 8. 


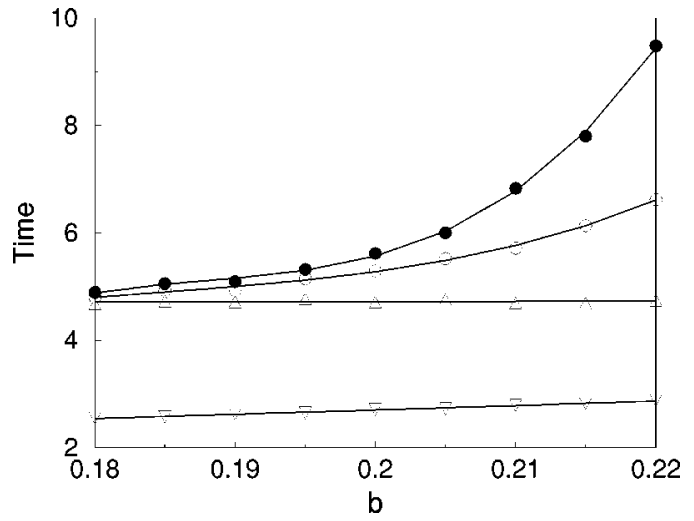

FIG. 11. Dependences of the characteristic turbulence time scale $T_{0}(\bigcirc)$, rotation period of spiral waves $(\bullet)$, recovery $(\triangle)$, and refractory $(\nabla)$ times on the parameter $b$ for $a=1.1$. Other paramers as in Fig. 1.

scrolls or scroll rings, when the boundary between the regions CS and ES in the parameter plane (Fig. 1) is crossed toward a lower excitability. Propagation of individual excitation waves or of wave trains is not at all distorted when this boundary is crossed. This means, for example, that the medium can still possess stable local wave sources (pacemakers) in the region ES. Nothing in their behavior would indicate that a boundary to the dangerous region has already been crossed. However, if a scroll wave is created in the medium, it would quickly give rise to turbulence in this region, which would correspond to fibrillation. In contrast to this, the scroll waves remain straight and the scroll rings shrink and disappear on the other side of the boundary, in the region $\mathrm{CS}$.

The simulations presented here have been done with a generic model of excitable media, but further numerical investigations with realistic models of the cardiac tissue are needed. Recently, evolution of scroll rings has been observed in the chemical Belousov-Zhabotinsky medium, using gels to decrease the inhibitor diffusion [49]. Similar experiments
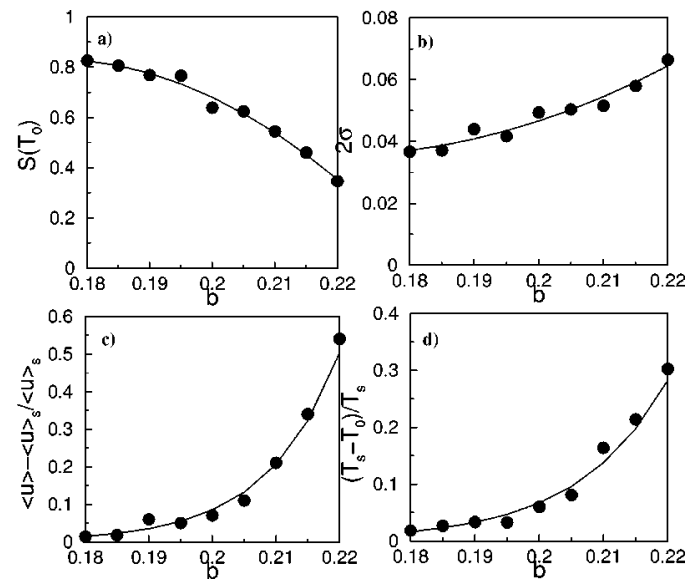

FIG. 12. Dependence of characteristic properties of negative tension turbulence on the parameter $b$ at a fixed $a=1.1$ : (a) the height $S\left(T_{0}\right)$ of the main maximum of the temporal correlation function, (b) the width $2 \sigma$ of the main power spectrum maximum, (c) the difference between the mean activation levels $\langle u\rangle$ and $\langle u\rangle_{s}$ for the turbulent state and for a spiral waves, and (d) the difference between the characteristic time scale $T_{0}$ of turbulence and the rotation period $T_{s}$ of spiral waves. Other paramers as in Fig. 1.

may provide a way for direct experimental observation of Winfree turbulence in chemical systems.

The scenario of wave turbulence here analyzed can be controlled (suppressed or induced) by appropriate nonresonant periodic modulation of the medium parameters [19]. A detailed account of our numerical investigations and analytical studies, related to such a control strategy, shall be a subject of a separate publication [30].

\section{ACKNOWLEDGMENTS}

This work was supported by Dirección General de Investigación (DGI) and Comissionat per a Universitat i Recerca (Generalitat de Catalunya) under Project Nos. BXX20000638 and 2001SGR00045.
[1] A. N. Zaikin and A. M. Zhabotinsky, Nature (London) 255, 535 (1970).

[2] F. Siegert and C. J. Weijer, Physica D 49, 224 (1991).

[3] J. M. Davidenko, A. V. Pertsov, R. Salomonsz, W. Baxter, and J. Jalife, Nature (London) 355, 349 (1992).

[4] A. T. Winfree, Chaos 8, 1 (1998).

[5] R. H. Clayton and A. V. Holden, Chaos, Solitons Fractals 13, 1579 (2002).

[6] D. J. Christini and L. Glass, Chaos 12, 732 (2002).

[7] A. T. Winfree, Science 181, 937 (1973).

[8] R. A. Gray et al., Science 270, 1222 (1995).

[9] V. N. Biktashev, A. V. Holden, and H. Zhang, Philos. Trans. R. Soc. London, Ser. A 347, 611 (1994).

[10] V. I. Krinsky, Problemy Kibernetiky (Nauka, Moskow, 1968) Vol. 20, p. 59.
[11] A. V. Panfilov and J. P. Keener, J. Theor. Biol. 163, 439 (1993).

[12] A. V. Panfilov and A. Holden, Phys. Lett. A 151, 23 (1990).

[13] M. Courtemanche and A. T. Winfree, Int. J. Bifurcation Chaos Appl. Sci. Eng. 1, 431 (1991).

[14] A. Karma, Phys. Rev. Lett. 71, 1103 (1993).

[15] M. Bär and M. Eiswirth, Phys. Rev. E 48, R1635 (1993).

[16] A. S. Mikhailov, Foundations of Synergetics I. Distributed Active Systems (Springer, Berlin, 1990).

[17] A. V. Panfilov and P. Hogeweg, Phys. Rev. E 53, 1740 (1996).

[18] A. T. Winfree, Science 266, 1003 (1994).

[19] S. Alonso, F. Sagués, and A. S. Mikhailov, Science 299, 1722 (2003).

[20] J. P. Keener, Physica D 31, 269 (1988).

[21] J. P. Keener and J. J. Tyson, Science 239, 1284 (1988). 
[22] A. V. Panfilov and A. N. Rudenko, Physica D 28, 215 (1987).

[23] P. K. Brazhnik, V. A. Davydov, V. S. Zykov, and A. S. Mikhailov, Sov. Phys. JETP 66, 984 (1987).

[24] V. N. Biktashev, Int. J. Bifurcation Chaos Appl. Sci. Eng. 8, 677 (1998).

[25] H. Henry and V. Hakim, Phys. Rev. Lett. 85, 5328 (2000).

[26] H. Henry and V. Hakim, Phys. Rev. E 65, 046235 (2002).

[27] F. H. Fenton et al., Chaos 12, 852 (2002).

[28] R. A. Gray and J. Jalife, Chaos 8, 65 (1998).

[29] F. Chávez, R. Kapral, G. Rousseau, and L. Glass, Chaos 11, 757 (2001).

[30] S. Alonso, F. Sagués and A. S. Mikhailov (unpublished).

[31] D. Barkley, M. Kness, and L. S. Tuckerman, Phys. Rev. A 42, 2489 (1990).

[32] I. Aranson and I. Mitkov, Phys. Rev. E 58, 4556 (1998).

[33] T. Amemiya, P. Kettunen, S. Kádár, T. Yamaguchi, and K. Showalter, Chaos 8, 872 (1998).

[34] F. Fenton and A. Karma, Phys. Rev. Lett. 81, 481 (1998).

[35] M. Vinson and A. Pertsov, Phys. Rev. E 59, 2764 (1999).

[36] R. M. Mantel and D. Barkley, Physica D 107, 149 (2001).

[37] V. Krinsky, E. Hamm, and V. Voignier, Phys. Rev. Lett. 76, 3854 (1996).

[38] A. V. Panfilov, A. N. Rudenko, and V. I. Krinsky, Biofizika 31, 323 (1986).

[39] A. S. Mikhailov, Chaos, Solitons Fractals 5, 673 (1995).

[40] A. T. Winfree, SIAM Rev. 32, 1 (1990).

[41] H. Henry, Phys. Rev. E 70, 026204 (2004).
[42] T. Yamaguchi, L. Kuhnert, Zs. Nagy-Ungvaray, S. C. Muller, and B. Hess, J. Phys. Chem. 95, 5831 (1991).

[43] W. Jahnke and A. T. Winfree, Int. J. Bifurcation Chaos Appl. Sci. Eng. 1, 891 (1991).

[44] See EPAPS Document No. E-PLEEE8-70-158409 for the complete movie of the irregular wave pattern evolution. A direct link to this document may be found in the online article's HTML reference section. The document may also be reached via The EPAPS homepage (http://www.aip.org/pubservs/ epaps.html) or from ftp.aip.org in the directory /epaps/. See The EPAPS homepage for more information.

[45] See EPAPS Document No. E-PLEEE8-70-158409 for the complete movie of the irregular filament evolution. A direct link to this document may be found in the online article's HTML reference section. The document may also be reached via The EPAPS homepage (http://www.aip.org/pubservs/epaps.html) or from ftp.aip.org in the directory /epaps/. See The EPAPS homepage for more information.

[46] M.-A. Bray and J. P. Wikswo, Phys. Rev. Lett. 90, 238303 (2003).

[47] B. Fiedler and R. M. Mantel, Doc. Math. 5, 695 (2000).

[48] F. X. Witkowski, L. J. Leon, P. A. Penkoske, W. R. Giles, M. L. Spano, W. L. Ditto, and A. T. Winfree, Nature (London) 392, 78 (1998).

[49] U. Storb, C. R. Neto, M. Bar, and S. C. Muller, Phys. Chem. Chem. Phys. 5, 2344 (2003). 